\title{
Copy number alterations and allelic ratio in relation to recurrence of rectal cancer
}

\author{
Inès J Goossens-Beumer ${ }^{1}$, Jan Oosting ${ }^{2}$, Wim E Corver ${ }^{2}$, Marjolein JFW Janssen ${ }^{3}$, Bart Janssen ${ }^{3}$, Wilbert van Workum ${ }^{3}$, \\ Eliane CM Zeestraten ${ }^{1}$, Cornelis JH van de Velde ${ }^{1}$, Hans Morreau ${ }^{2}$, Peter JK Kuppen ${ }^{1}$ and Tom van Wezel ${ }^{2^{*}}$
}

\begin{abstract}
Background: In rectal cancer, total mesorectal excision surgery combined with preoperative (chemo)radiotherapy reduces local recurrence rates but does not improve overall patient survival, a result that may be due to the harmful side effects and/or co-morbidity of preoperative treatment. New biomarkers are needed to facilitate identification of rectal cancer patients at high risk for local recurrent disease. This would allow for preoperative (chemo)radiotherapy to be restricted to high-risk patients, thereby reducing overtreatment and allowing personalized treatment protocols. We analyzed genome-wide DNA copy number (CN) and allelic alterations in 112 tumors from preoperatively untreated rectal cancer patients. Sixty-six patients with local and/or distant recurrent disease were compared to matched controls without recurrence. Results were validated in a second cohort of tumors from 95 matched rectal cancer patients. Additionally, we performed a meta-analysis that included 42 studies reporting on $\mathrm{CN}$ alterations in colorectal cancer and compared results to our own data.

Results: The genomic profiles in our study were comparable to other rectal cancer studies. Results of the meta-analysis supported the hypothesis that colon cancer and rectal cancer may be distinct disease entities. In our discovery patient study cohort, allelic retention of chromosome 7 was significantly associated with local recurrent disease. Data from the validation cohort were supportive, albeit not statistically significant, of this finding.
\end{abstract}

Conclusions: We showed that retention of heterozygosity on chromosome 7 may be associated with local recurrence in rectal cancer. Further research is warranted to elucidate the mechanisms and effect of retention of chromosome 7 on the development of local recurrent disease in rectal cancer.

\section{Background}

The Dutch Total Mesorectal Excision (TME) trial [1] changed standard treatment guidelines for rectal cancer patients [2]. This international trial was designed to provide a clinical assessment of whether additional preoperative short-term radiotherapy (pRT) could reduce the number of local recurrences compared to TME surgery alone. Based on the Dutch TME trial, patients with tumor stage 2 (T2) were recommended to receive pRT in addition to TME surgery [2]. The beneficial effect of TME surgery combined with pRT on rectal cancer local recurrence rates was subsequently confirmed in a Dutch population-based study [3]. In addition to pRT, preoperative concurrent chemoradiation treatment

\footnotetext{
* Correspondence: T.van_Wezel@lumc.nl

${ }^{2}$ Department of Pathology, L1-Q, Leiden University Medical Center, PO Box 9600, 2300 RC, Leiden, The Netherlands

Full list of author information is available at the end of the article
}

(pCCRT) has been studied internationally and proposed as a preoperative treatment for the reduction of local recurrent disease related to rectal cancer. Reductions in local recurrence achieved by pCCRT were similar to those for pRT [4], and the postoperative complications associated with pRT and pCCRT were also reported to be comparable [5]. In 2013, an EURECCA consensus was published which provided treatment recommendations for rectal cancer aimed at minimizing the differences in rectal cancer treatment regimes in Europe $[6,7]$. Although both pRT and pCCRT are recommended for specific TNM stages and introduction of these approaches has reduced local recurrence rates markedly, no difference in overall survival has yet been achieved $[2,4,8]$. This lack of survival benefit is most likely caused by the harmful side effects [9] and comorbidity of the preoperative treatment [2]. Recently, a 
phase III clinical trial named RAPIDO was initiated to assess the survival benefit of pRT followed by full-dose preoperative chemotherapy as an alternative for PCCRT with and without postoperative chemotherapy [10].

Under current guidelines, preoperative treatment is offered to a broad group of patients, although only around $10 \%$ of patients are actually at risk for development of a local recurrence without preoperative treatment [2]. This means that up to $90 \%$ of rectal cancer patients are unnecessarily exposed to preoperative treatment [2]. This undesirable situation calls for effective biomarkers that allow for selection of those patients with a high probability for development of a local recurrence after surgery alone. Effective selection for pRT or pCCRT treatment of only those rectal cancer patients with an increased risk of recurrent local disease would reduce overtreatment and allow for more personalized treatment protocols.

Genetic profiling of copy number $(\mathrm{CN})$ alterations in individual chromosomes has previously been recognized as an independent predictor for metastatic relapse of early stage colorectal cancer [11]. The risk of development of a local recurrence in rectal cancer can be deduced from the genetic aberrations found in the primary tumor. In the current study, we were especially interested in the prognostic value of genomic alterations related to local recurrent disease in rectal cancer. The aim of our study was therefore to identify $\mathrm{CN}$ alterations and allelic imbalances that could predict risk of recurrence in rectal cancer. Using a genome-wide $\mathrm{CN}$ analysis approach, we analyzed tissues from 112 rectal cancer patients enrolled in the TME trial. High-density SNP arrays were used to assess $\mathrm{CN}$ alterations and allelic (im)balances of genomic DNA (gDNA) segments [12,13]. As many studies have reported on $\mathrm{CN}$ alterations in (colo)rectal cancer, we additionally performed a metaanalysis of 42 studies that reported chromosomal $\mathrm{CN}$ alterations (high frequency $\mathrm{CN}$ alterations at the level of chromosome arms) and compared these to our current findings. We hypothesized that the $\mathrm{CN}$ alterations and/or aberrant allelic ratio patterns of certain gDNA segments might be prognostic for a local recurrence in rectal cancer, and might therefore identify those patients that would benefit most from pRT or pCCRT.

\section{Study cohort}

Tumor and matching normal tissues from the Dutch TME trial, which recruited rectal cancer patients in 118 European centers and one Canadian center, were available for analysis. The trial design has been described previously [1]. We selected patients (from the non-radiation treatment arm) with clinically resected TNM stage I-III adenocarcinomas of the rectum and a presentation of local and/or distant recurrent disease at follow-up. To exclude uncontrolled bias, patients with clinically resected TNM stage I-III adenocarcinomas of the rectum presenting without recurrent disease were additionally selected to match those presenting with recurrent disease. Individual control group patients matched patients in the local recurrence group, the distant recurrence group and the local \& distant recurrence group. For every patient within a recurrence group (local, distant, or local \& distant) a unique match was therefore included. Matching criteria were TNM stage (exact match), CRM involvement (exact match), gender (exact match), and age at surgery (average difference of 2 years; range 0-7 years).

For the discovery phase, we selected tissues from 112 rectal cancer patients for whom fresh-frozen (FF) tumor and normal matched tissues were available. These included samples from patients with a recurrence (all available local $\mathrm{N}=10$, distant $\mathrm{N}=41$, and all available local \& distant $\mathrm{N}=15$ ) and matched control patients without a recurrence $(\mathrm{N}=46)$. Three samples from the discovery cohort were used for technical validation. For validation of results, we selected tumor and normal matched formalin-fixed paraffin-embedded (FFPE) tissue from 95 patients. Patients presenting with a recurrence (all available local, $\mathrm{N}=12$, distant, $\mathrm{N}=24$, and all available local \& distant, $\mathrm{N}=22$ ) were matched to controls (without any recurrence, $\mathrm{N}=37$ ). The discovery study cohort $(\mathrm{N}=112)$ and validation cohort $(\mathrm{N}=95)$, which did not overlap, are described in Table 1.

\section{Consent}

All samples were coded in accordance with national ethical guidelines ("Code for Proper Secondary Use of Human Tissue", Dutch Federation of Medical Scientific Societies). The use of these specimens was approved by the Medical Ethical Committee of the Leiden University Medical Center (LUMC). Patients were included in The Dutch Total Mesorectal Excision (TME) trial after informed consent was obtained [1].

\section{DNA isolation}

Genomic DNA (gDNA) from tumor and corresponding normal tissue (FF or FFPE) was isolated, as described previously [14,15], following macrodissection or laser capture microdissection (LCM). The DNA concentration was measured using the PicoGreen method (Invitrogen, Carlsbad, CA, USA). If necessary, samples were concentrated using a Speedvac (SC110A, ThermoFisher, Waltham, MA, USA) to $10 \mathrm{ng} / \mu \mathrm{l}$ or to a minimum of $15 \mu \mathrm{l}$.

\section{CytoSNP arrays and analysis}

Hybridization of gDNA to high resolution Illumina Human CytoSNP12v2 arrays (Illumina, San Diego, CA, USA), intensity data extraction and the first quality control steps were performed by ServiceXS (Leiden, the 
Table 1 Summary of rectal cancer study cohorts

\begin{tabular}{|c|c|c|c|c|}
\hline & \multicolumn{2}{|c|}{ Discovery cohort (FF) } & \multicolumn{2}{|c|}{ Validation cohort (FFPE) } \\
\hline & $\mathrm{N}=112$ & Perc. & $\mathrm{N}=95$ & Perc. \\
\hline \multicolumn{5}{|l|}{ Age at surgery } \\
\hline$<50$ & 14 & $13 \%$ & 4 & $4 \%$ \\
\hline $50-75$ & 72 & $64 \%$ & 72 & $76 \%$ \\
\hline$\geq 75$ & 26 & $23 \%$ & 19 & $20 \%$ \\
\hline \multicolumn{5}{|l|}{ Gender } \\
\hline Male & 73 & $65 \%$ & 57 & $60 \%$ \\
\hline Female & 39 & $35 \%$ & 38 & $40 \%$ \\
\hline \multicolumn{5}{|l|}{ TNM stage } \\
\hline Stage I & 11 & $10 \%$ & 7 & $7 \%$ \\
\hline Stage II & 24 & $21 \%$ & 21 & $22 \%$ \\
\hline Stage III & 77 & $69 \%$ & 67 & $71 \%$ \\
\hline \multicolumn{5}{|l|}{ CRM involvement } \\
\hline No & 82 & $73 \%$ & 56 & $59 \%$ \\
\hline Yes & 30 & $27 \%$ & 39 & $41 \%$ \\
\hline \multicolumn{5}{|l|}{ Recurrence } \\
\hline No recurrence & 46 & $41 \%$ & 37 & $39 \%$ \\
\hline Local $^{a, b}$ & 10 & $9 \%$ & 12 & $13 \%$ \\
\hline Distant $^{\mathrm{b}}$ & 41 & $37 \%$ & 24 & $25 \%$ \\
\hline Local \& Distant ${ }^{a, b}$ & 15 & $13 \%$ & 22 & $23 \%$ \\
\hline
\end{tabular}

Abbreviations: ${ }^{\mathrm{a}} \mathrm{All}$ available patients with fresh frozen/FFPE tumor and normal tissue specimens were included; ${ }^{b}$ Patients with a recurrence were matched to patients without a recurrence based on the matching criteria TNM stage, CRM involvement, gender, and age at surgery; $\mathrm{N}=$ Number of patients; Perc. $=$ Percentage of total patients.

Netherlands). Bioinformatic analysis was performed using the beadarraySNP package in $\mathrm{R}$ [13]. After normalization, de-waving and automated segmentation analysis, we obtained $\mathrm{CN}$ data and allelic ratio data for all arrays. Thresholds for $\mathrm{CN}$ alterations after normalization were set at 0.92 for losses and 1.08 for gains, compared to the sample mean. To designate the allelic ratio groups, DNA segments were divided per individual sample into three classes, retention of heterozygosity, imbalance and loss of heterozygosity ( $\mathrm{LOH})$.

A globaltest ( $\mathrm{R}$ package globaltest $[16,17])$ was performed on both the continuous $\mathrm{CN}$ data and the overall allelic ratio group data separately to determine overall statistical differences between analysis groups, comparing local recurrence group (L-group) versus control group (C-group), distant recurrence group (D-group) versus C-group and local \& distant recurrence group (LD-group) versus C-group. A significant global test on the overall $\mathrm{CN}$ data or allelic ratio group data resulted in a second global test on individual chromosome arms. If significant, the smaller underlying segments underwent a further global test to identify regions with significant differences between the analysis groups. Multiple testing correction was performed using the Benjamin-Hochberg (BH) method [18].

For allelic ratio group analyses of chromosome combinations, the overall chromosome status was classified into balanced $=1$, imbalanced $=2$ and $\mathrm{LOH}=3$. Overall chromosome status was defined as most abundant allelic ratio group number on the chromosome. In the chromosome combination analyses, the highest number of overall chromosome status was used. Differences between groups were assessed with Fisher's exact test for count data.

\section{Survival analysis}

Survival analysis was performed using the Cox proportional hazards model (R-package survival). Survival data were available for 12 years of follow-up. The mean follow-up time of the discovery cohort and the validation cohort were 6.6 years (range 0.07-13.4 years) and 5.7 years (range 0.0913.6 years), respectively. Overall survival (OS) was defined as the time from surgery until death by any cause. Disease specific survival (DSS) was defined as the time from surgery until death by rectal cancer. Local recurrence-free period (LRFP) was defined as the time from surgery until the discovery of a local recurrence. Distant recurrence-free period (DRFP) was defined as the time from surgery until the discovery of a distant recurrence. Multivariate models included the predetermined clinically important covariates TNM stage, age at surgery, gender and circumferential margin involvement, irrespective of statistical significance.

\section{Dynamic array and analysis}

Based on the results from the discovery phase, reference SNP (rs) identification numbers on chromosome 7 and 13 were extracted from the CytoSNP12 array. A search was performed for validated ABI-Taqman SNP assays, based on rs-numbers, in the SNP browser program (Applied Biosystems, Foster City, CA, USA) and 48 SNPs were selected for validation, with 16 on chromosome 7p, 16 on chromosome 7q and 16 on chromosome 13 (Additional file 1 S1). Selection was based on the highest percentage of heterozygosity (at least $40 \%$ ) as determined in the genotype call for normal tissues $(\mathrm{N}=112)$ in the discovery phase.

Taqman assays targeting the 48 selected SNPs were tested using the 96.96 BioMark Dynamic Array for quantitative Real-Time PCR (Fluidigm Corporation, San Francisco, CA, USA). On the 96.96 BioMark dynamic platform, Taqman SNP targets were assayed in duplicate and samples were assayed in triplicate (technical validation) or duplicate (validation) using 14 cycles of Specific Target Amplification for each sample replicate prior to the qPCR on the array. Assays were performed by ServiceXS. Fluidigm Real-Time PCR analysis software was used to extract cycle threshold $(\mathrm{Ct})$ values, while Fluidigm SNP Genotyping analysis software was 
used to extract endpoint measurements at cycle 40 and for genotype calling. The linear derivative was used to determine $\mathrm{Ct}$ threshold values per SNP assay. Samples with a $\mathrm{Ct}>30$ and missing values were set at $\mathrm{Ct}=30$. The number of starting DNA molecules per reaction chamber was computed using the following formula: \# DNA molecules $=2^{\wedge}(26-\mathrm{Ct})$. In the 96.96 BioMark dynamic assay, a $\mathrm{Ct}$ value of 26 corresponds to one DNA molecule in the reaction chamber. Total sample molecule amount of the VIC (A-allele) and FAM (B-allele) channels was used for sample normalization. The allelic ratio, based on calculated amounts of DNA molecules rather than intensity, can be calculated by dividing the values for the VIC (A-allele) and FAM (B-allele) channels.

\section{Validation of results}

Based on quality control, samples with a mean log2 value lower than 4, and patients with non-matching tumor and normal SNP genotypes, were excluded from analyses. The Welch Two Sample T-test was used to assess whether average allelic ratio values along chromosome $7 \mathrm{p}$, chromosome $7 \mathrm{q}$ or chromosome 13 were significantly different between the recurrence groups and the control group. Survival analysis for validation of results was performed as described above.

\section{Results and discussion}

\section{Description of frequently occurring copy number alterations, and meta-analysis}

We assessed $\mathrm{CN}$ alterations and chromosomal aberrations in rectal cancers from the Dutch TME trial, selected from the treatment arm that did not receive pRT. To gain a broader perspective on the results of our analysis of chromosomal $\mathrm{CN}$ alterations, we performed a meta-analysis of previously published $\mathrm{CN}$ studies. A PubMed search (performed on April 7, 2014), using the search criteria described in Additional file 2 S2, yielded 325 published studies. Studies from 2005 to search date and additional studies (selected from references of the selected articles published in 2005 or after) that described older $\mathrm{CN}$ alteration studies were assessed for eligibility, which required description of at least 6 tumors and detailed information on $\mathrm{CN}$ alterations per chromosome arm. A total of 42 studies that reported data on $\mathrm{CN}$ alterations in rectal cancer or colorectal cancer were included [11,19-59]. We combined results from these 42 studies (Table 2) and one additional study published online just after our Pubmed search (Table 2) and used the data to locate high frequency $\mathrm{CN}$ alterations in colorectal cancer at the level of chromosome arms (Figure 1A). CN alterations reported in at least $40 \%$ of the studies for at least
$25 \%$ of the study cases were considered to be common $\mathrm{CN}$ alterations with high frequencies.

Many included studies pooled colon and rectal tumors and referred to them as colorectal cancer. However, evidence suggests that tumors arising from colon and rectal tissues should be considered as distinct disease entities [60-62]. We therefore compared common $\mathrm{CN}$ alterations with high frequencies in rectal versus colon cancers. Twenty studies (including the present study) specifically reported $\mathrm{CN}$ alterations in rectal carcinomas (Figure 1B). Frequent gains common in rectal cancer included regions of chromosomes $7 \mathrm{p}$ (found in 75\% of the 20 rectal cancer studies, with a frequency $>25 \%), 7 q$ (65\%), 8q (100\%), 13q (100\%), 20p (70\%) and 20q (85\%). Frequent losses common in rectal cancer were found on chromosomes $1 \mathrm{p}$ (found in $55 \%$ of the 20 rectal cancer studies, with a frequency $>25 \%), 4 q(50 \%), 5 q(40 \%), 8 p$ (80\%), 14q (40\%), 15q (65\%), 17p (95\%), 18p (70\%), 18q (100\%) and 22q (50\%). Twelve studies reported $\mathrm{CN}$ alterations specific to colon carcinomas (Figure 1C), with frequent gains common in colon cancer on chromosomes $7 \mathrm{p}$ (found in $67 \%$ of the 12 studies, with a frequency $>25 \%$ ), 7q (75\%), 8q (75\%), 13q (83\%), 20p (42\%) and 20q (92\%). Frequent losses common in colon cancer were found on chromosomes $8 \mathrm{p}$ (found in $42 \%$ of the 12 studies, with a frequency $>25 \%), 17 p(67 \%), 18 p(67 \%)$ and $18 q(92 \%)$. In contrast to rectal cancer, frequent losses on chromosomes $1 \mathrm{p}, 4 \mathrm{q}, 5 \mathrm{q}, 14 \mathrm{q}, 15 \mathrm{q}$ and $22 \mathrm{q}$ were not observed in at least $40 \%$ of the colon studies. With the exception of loss at $4 q$, these chromosome arms showed a difference of $>20 \%$ between rectal cancer and colon cancer studies, indicating that rectal cancers were associated with a higher frequency of losses on these particular chromosome arms (Figure 1D). In addition, the percentage of studies reporting frequent losses on chromosomes $1 \mathrm{q}, 8 \mathrm{p}, 17 \mathrm{p}$, and $21 \mathrm{q}$, and the percentage of studies reporting frequent gains on chromosomes $8 \mathrm{q}$ and 20p, was higher in rectal cancer compared to colon cancer. These differences may be explained, in part, by the much higher percentage of microsatellite unstable colon carcinomas in comparison to rectal carcinomas, because microsatellite instability reflects DNA mismatch repair deficiency, in contrast to chromosomal instability characterized by aneuploidy. Where possible, microsatellite unstable tumors were omitted from our comparisons, although many studies did not report microsatellite stability status.

In conclusion, frequent $\mathrm{CN}$ alterations common in rectal cancer were located on chromosomes $1 \mathrm{p}, 4 \mathrm{q}, 5 \mathrm{q}$, $7,8,13 q, 14 q, 15 q, 17 p, 18,20$ and 22q, while colon cancer showed a similar pattern but with a lower frequency of gains at chromosomes $8 \mathrm{q}$ and $20 \mathrm{p}$, a lower frequency of losses at chromosomes 1q, 8p, 17p and 21q, and an absence of frequent $\mathrm{CN}$ losses at chromosomes $1 \mathrm{p}, 4 \mathrm{q}$, $5 \mathrm{q}, 14 \mathrm{q}, 15 \mathrm{q}$ and $22 \mathrm{q}$. 
Table 2 Colorectal cancer studies reporting on copy number alterations

\begin{tabular}{|c|c|c|c|c|c|c|c|c|c|c|c|}
\hline & \multirow[t]{2}{*}{ Studies } & & \multirow[t]{2}{*}{ Origin of tissues } & \multirow[t]{2}{*}{ Method } & \multicolumn{2}{|l|}{ Total CRC } & \multicolumn{2}{|l|}{ Rectum } & \multicolumn{2}{|l|}{ Colon } & \multirow{2}{*}{$\begin{array}{l}\text { TNM } \\
\text { stage }\end{array}$} \\
\hline & & & & & Figure $1 \mathrm{~A}$ & $\#$ & Figure 1B & $\#$ & Figure 1C & $\#$ & \\
\hline 1 & Schlegel et al. & 1995 & Germany & $\mathrm{CGH}$ & yes & 12 & no & $(x)$ & yes & 12 & \\
\hline 2 & Ried et al. & 1996 & Germany & $\mathrm{CGH}$ & yes & 16 & yes & 6 & yes & 10 & $2 / 3 /(4)$ \\
\hline 3 & Meijer et al. & 1998 & the Netherlands & $\mathrm{CGH}$ & yes & 14 & yes & 7 & yes & 7 & \\
\hline 4 & Nakao et al. & 1998 & Japan & $\mathrm{CGH}$ & yes & 9 & no & 0 & yes & 9 & $(1) / 3$ \\
\hline 5 & Paredes-Zaglul et al. & 1998 & USA & $\mathrm{CGH}$ & yes & 9 & yes & 2 & yes & 7 & (3)/4 \\
\hline 6 & Al-Mulla et al. & 1999 & UK & $\mathrm{CGH}$ & yes & 12 & no & $(x)$ & no & $(x)$ & $2 / 3$ \\
\hline 7 & De Angelis et al. & 1999 & Norway & $\mathrm{CGH}$ & yes & 45 & no & $(x)$ & no & $(x)$ & $2 / 3 / 4$ \\
\hline 8 & Georgiades et al. & 1999 & UK & $\mathrm{CGH}$ & yes & 17 & no & $(11)$ & no & (6) & $1 / 2 / 3$ \\
\hline 9 & Korn et al. & 1999 & USA & $\mathrm{CGH}$ & yes & 6 & no & (0) & yes & 6 & $2 / 3 / 4$ \\
\hline 10 & Aust et al. & 2000 & USA/Germany & $\mathrm{CGH}$ & yes & 42 & no & (7) & no & (35) & $1 / 2 / 3 / 4$ \\
\hline 11 & Aragane et al. & 2001 & Japan & $\mathrm{CGH}$ & yes & 30 & no & $(x)$ & no & $(x)$ & $1 / 2 / 3 / 4$ \\
\hline 12 & Chan et al. & 2001 & China/UK & $\mathrm{CGH}$ & yes & 16 & no & $(x)$ & no & $(x)$ & $2 / 3 / 4$ \\
\hline 13 & De Angelis et al. & 2001 & Norway & $\mathrm{CGH}$ & yes & 67 & no & $(x)$ & no & $(x)$ & $1 / 2 / 3 / 4$ \\
\hline 14 & Nakao et al. & 2001 & Japan & $\mathrm{CGH}$ & yes & 35 & no & (13) & no & $(22)$ & $2 / 3 / 4$ \\
\hline 15 & Rooney et al. & 2001 & USA & $\mathrm{CGH}$ & yes & 29 & no & (4) & no & $(25)$ & 3 \\
\hline 16 & Hermsen et al. & 2002 & the Netherlands & $\mathrm{CGH}$ & yes & 82 & no & $(x)$ & no & $(x)$ & $1 /(2)$ \\
\hline 17 & Knösel et al. & 2002 & Germany & $\mathrm{CGH}$ & yes & 15 & no & $(x)$ & no & $(x)$ & \\
\hline 18 & Alcock et al. & 2003 & UK & $\mathrm{CGH}$ & yes & 17 & yes & 6 & yes & 11 & \\
\hline 19 & Ghadimi et al. & 2003 & Germany & $\mathrm{CGH}$ & yes & 50 & yes & 18 & yes & 32 & $1 / 2 / 3$ \\
\hline 20 & He et al. & 2003 & China & $\mathrm{CGH}$ & yes & 26 & yes & 14 & yes & 12 & $2 / 3 / 4$ \\
\hline 21 & Leslie et al. & 2003 & UK & $\mathrm{CGH}$ & yes & 50 & no & $(21)$ & no & (29) & $1 / 2 / 3$ \\
\hline 22 & Bardi et al. & 2004 & Sweden/Denmark & $\mathrm{CGH}$ & yes & 115 & no & $(51)$ & no & $(63)$ & $1 / 2 / 3 / 4$ \\
\hline 23 & Diep et al. & 2004 & Norway/Sweden & $\mathrm{CGH}$ & yes & 10 & no & $(x)$ & no & $(x)$ & (3)/4 \\
\hline 24 & Nakao et al. & 2004 & Spain & $\mathrm{aCGH}$ & yes & 125 & no & $(x)$ & no & $(x)$ & $1 / 2 / 3 / 4$ \\
\hline 25 & Poeaim et al. & 2005 & Thailand & $\mathrm{CGH}$ & yes & 40 & no & $(10)$ & no & (30) & \\
\hline 26 & Tanami et al. & 2005 & Japan & $\mathrm{CGH}$ & yes & 20 & yes & 9 & yes & 11 & $(2) /(3) / 4$ \\
\hline 27 & Al-Mulla et al. & 2006 & UK/Kuwait & $\mathrm{CGH}$ & yes & 70 & no & $(47)$ & no & (18) & $1 / 2$ \\
\hline 28 & Grade et al. & 2006 & Germany & $\mathrm{CGH}$ & yes & 21 & yes & 21 & no & 0 & $2 / 3$ \\
\hline 29 & Grade et al. ${ }^{a}$ & 2007 & Germany & $\mathrm{CGH}$ & yes & 32 & no & 0 & yes & 32 & $2 / 3$ \\
\hline 30 & Lips et al. & 2007 & the Netherlands & $\mathrm{SNPa}$ & yes & 77 & yes & 77 & no & 0 & $1 / 2 / 3$ \\
\hline 31 & Xiao et al. & 2007 & China & $\mathrm{CGH}$ & yes & 24 & yes & 9 & yes & 15 & \\
\hline 32 & Lips et al. & 2008 & the Netherlands & SNPa & yes & 32 & yes & 32 & no & 0 & $1 / 2 /(3)$ \\
\hline 33 & Grade et al. ${ }^{a}$ & 2009 & Germany & $\mathrm{CGH}$ & yes & 42 & yes & 42 & no & 0 & $1 / 2 / 3$ \\
\hline 34 & Lagerstedt et al. & 2010 & Sweden & $\mathrm{aCGH}$ & yes & 24 & no & $(\mathrm{x})$ & no & $(x)$ & $1 / 2 / 3 / 4$ \\
\hline 35 & Molinari et al. & 2011 & Italy & $\mathrm{aCGH}$ & yes & 51 & yes & 51 & no & 0 & $1 / 2 / 3$ \\
\hline 36 & Nakao et al. & 2011 & Japan & $\mathrm{aCGH}$ & yes & 94 & no & $(x)$ & no & $(x)$ & $1 / 2 / 3 / 4$ \\
\hline 37 & Chen et al. ${ }^{b}$ & $2011 / 2012$ & USA & $\mathrm{aCGH}$ & yes & 95 & yes & 95 & no & 0 & $2 / 3$ \\
\hline 38 & Kodeda et al. & 2012 & Sweden & $\mathrm{aCGH}$ & yes & 16 & yes & 16 & no & 0 & $1 / 2 / 3$ \\
\hline 39 & Shi et al. & 2012 & China & $\mathrm{aCGH}$ & yes & 8 & yes & 8 & no & 0 & \\
\hline 40 & Liang et al. & 2013 & China & $\mathrm{aCGH}$ & yes & 48 & yes & 48 & no & 0 & $2 / 3 / 4$ \\
\hline 41 & Zhou et al. & 2013 & China & $\mathrm{aCGH}$ & yes & 16 & yes & 16 & no & 0 & $2 / 3$ \\
\hline 42 & Doyen et al. & 2014 & France/Germany & SNPa & yes & 80 & yes & 80 & no & 0 & $(1 / 2 / 3)$ \\
\hline
\end{tabular}


Table 2 Colorectal cancer studies reporting on copy number alterations (Continued)

\begin{tabular}{|c|c|c|c|c|c|c|c|c|c|c|c|}
\hline \multirow[t]{2}{*}{43} & This study & 2014 & the Netherlands & $\mathrm{SNPa}$ & yes & 112 & yes & 112 & no & 0 & $1 / 2 / 3$ \\
\hline & Total & & & & 43 & & 20 & & 12 & & \\
\hline
\end{tabular}

This table lists the published studies on $\mathrm{CN}$ alterations in rectal carcinomas used for comparisons with the present study (Nr. 43).

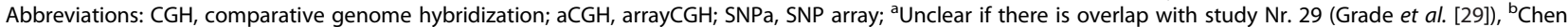
et al. published two papers based on the same patient cohort. Numbers in parentheses indicate the number of rectal or colon cancer patients included in the study, but for which results could not be distinguished between groups.

\section{Comparison of our study with the meta-analysis}

In order to identify $\mathrm{CN}$ alterations associated with a recurrence in rectal cancer, we assessed $\mathrm{CN}$ alterations in a series of non-preoperatively treated rectal cancers from the Dutch TME trial. Rectal cancers with local and/or distant recurrences were compared with matching control cases without recurrence. In terms of the location of frequent gains, our study cohort was comparable to previously described rectal cancer cohorts (Figure 1B upper panel). In contrast, compared to previous studies we detected a higher number of chromosome arms with frequent losses (Figure 1B lower panel). This might be due to our use of high-density arrays rather than CGH arrays, and to the selection of previously studied CGH locations. Another explanation might be the larger sample size of our study compared to most of the earlier studies, as smaller studies are more prone to higher variance. Additionally, our study differs from other studies due to
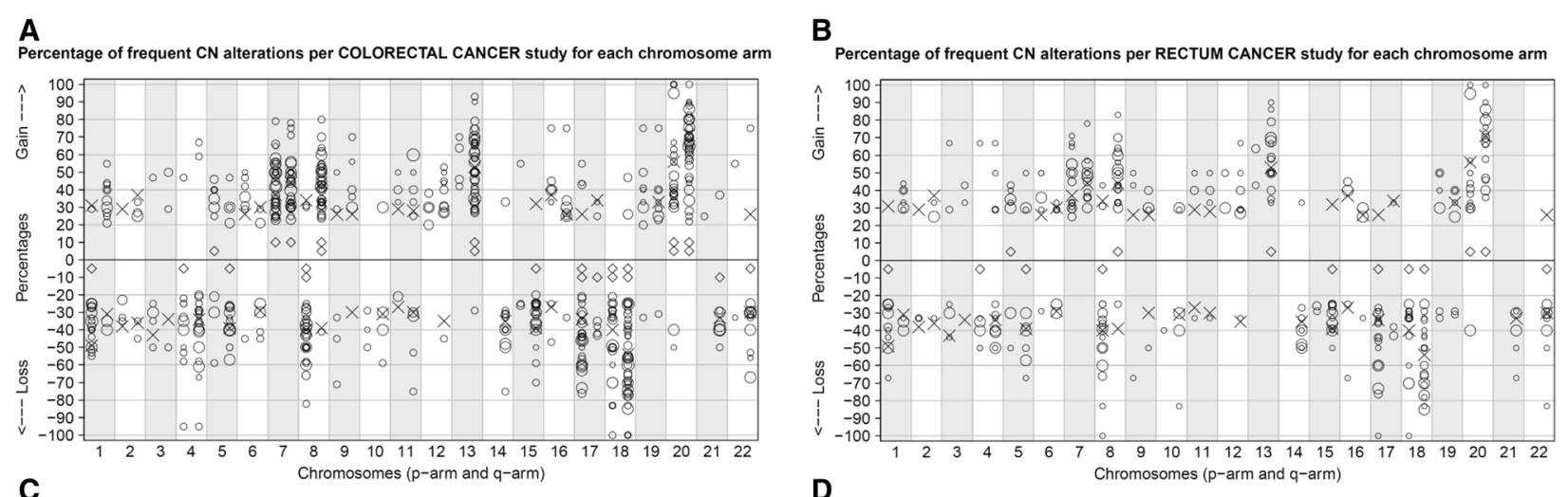

C

D
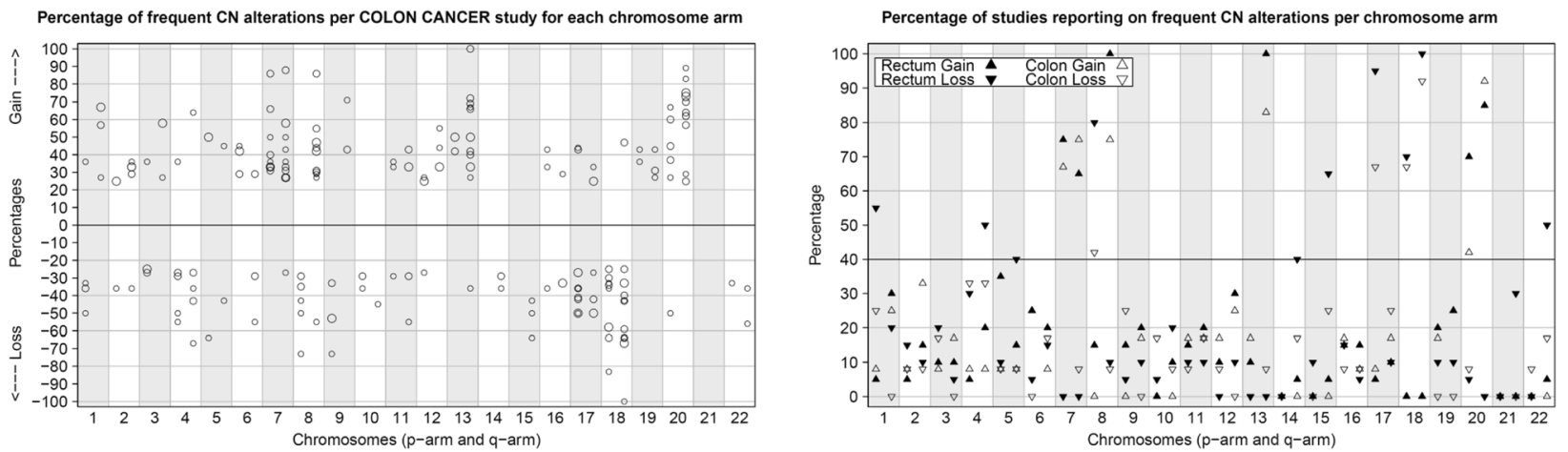

Figure 1 Frequent copy number alterations reported in colorectal cancer studies. Frequent CN alterations were defined as copy number (CN) changes found in at least $25 \%$ of the cases in a study cohort. Since most studies did not report low frequency CN alterations, only high frequency $\mathrm{CN}$ alterations were summarized. A-C. Percentage of high frequency $\mathrm{CN}$ alterations for each chromosome arm reported by different CGH/SNP studies for colorectal (A; plotted from 20\% to 100\%), rectum (B; plotted from 25\% to 100\%) or colon (C; plotted from 25\% to 100\%) cancer. Top panel: Percentages of gains. Lower panel; percentages of losses. Symbol size (circles/diamonds/X's) indicates the number of rectal cases included in a particular study. X indicates the CN percentage from the current (SNP) study. Indicated losses are restricted to segments containing at least 15 SNPs. Open circles $(\mathrm{O})$ represent other studies with reported percentages or percentages that could be estimated from plots or figures. For two studies [31,53], indicated by black diamonds $(\boldsymbol{\nabla})$, percentages were not available. $\mathbf{D}$. The percentage of studies, including the results of this study, were counted and plotted that reported on a chromosome arm showing CN alterations in $\geq 25 \%$ of the cases of that particular study. The percentages per chromosome arm for rectal studies are indicated with black-filled triangles $(\boldsymbol{\Lambda} \boldsymbol{\nabla})$, while colon studies are indicated by open triangles $(\Delta \nabla)$. Triangles that point upwards $(\Delta \Delta)$ indicate the number of studies with reported gains and triangles that point downwards $(\boldsymbol{\nabla} \nabla)$ indicate the number of studies reporting on losses found on the various chromosome arms. We considered a particular CN alteration to be common, with high frequency, when at least $40 \%$ of the studies reported the CN alteration in at least $25 \%$ of the study's cases. 
our use of both laser capture microdissection and macrodissection. This could have influenced results by reducing a potential effect in the analyses.

\section{Genomic abnormalities and local recurrence}

Continuous $\mathrm{CN}$ values and allelic ratio groups were analyzed in an effort to identify differentially affected genomic regions in rectal cancer patients presenting with and without local and/or distant recurrence. We included all patients with a local recurrence for whom fresh frozen tissue was available $(\mathrm{N}=25)$. Fifteen of these patients also presented with a distant recurrence. Additionally, we included 41 patients presenting with only distant recurrences. All patients with recurrent disease were matched to control samples of patients who did not present with a recurrence during follow-up $(\mathrm{N}=46)$.

The frequency of gains (Additional file $3 \mathrm{~S} 3 \mathrm{~A}$ ) and losses (Additional file $3 \mathrm{~S} 3 \mathrm{~B}$ ) along the length of each chromosome was plotted for each analysis group (control group, local recurrence group, distant recurrence group, and local \& distant recurrence group). $\mathrm{CN}$ gains were less frequently observed in the local recurrence group compared to the other analysis groups, while $\mathrm{CN}$ losses were more frequently observed in patients with a local recurrence. However, $\mathrm{CN}$ alterations were not significantly associated with (local) recurrent disease in our study cohort. A similar pattern can be deduced from a study by Diep et al. [49], with fewer $\mathrm{CN}$ gains and more $\mathrm{CN}$ losses in local recurrences compared to independent primary colorectal tumor samples. A lower frequency of gains along chromosome 7 and higher frequency of losses along chromosome 18q were especially associated with local recurrence in both studies. A study by Kodeda et al. [31], comparing locally recurrent and nonrecurrent rectal tumors, reported that both $\mathrm{CN}$ gains and $\mathrm{CN}$ losses were less frequently observed in locally recurrent tumors. Lower percentages of $\mathrm{CN}$ gains on chromosomes 8q, 13q and 20q, and a lower frequency of $\mathrm{CN}$ losses on chromosome 18q were observed in locally recurrent tumors in both our study cohort and the Kodeda cohort. In addition, we observed a lower frequency of $\mathrm{CN}$ gains on chromosomes 9p, 16p, 17q, 19 and $22 \mathrm{q}$ in the locally recurrent tumors. In contrast to the Kodeda study, we observed a higher frequency of $\mathrm{CN}$ losses on chromosomes $1 \mathrm{p}$ and $5 \mathrm{q}$ in locally recurrent tumors, in addition to a higher frequency of losses on chromosomes 3p, 9p and 15q. Overall, the results of the Kodeda study were comparable with our study regarding the lower frequency of $\mathrm{CN}$ gains, but conflicting on frequency of $\mathrm{CN}$ losses. This difference might be a result of the higher number of small deletions identified in our study due to our use of high-density SNP arrays compared to the $\mathrm{CGH}$ arrays used by Kodeda et al. However, despite these differences both the Kodeda study [31] and our study share a common conclusion that none of the above described $\mathrm{CN}$ alterations are (statistically) significantly associated with local recurrent disease.

An important feature of the SNP arrays used in our study was that they allow assessment of allelic imbalances, in addition to $\mathrm{CN}$ alterations. Using array data, allelic ratio groups were analyzed in order to identify genomic regions that showed allelic (im)balances associated with local and/or distant recurrence. The allelic ratio of DNA segments was classified into three classes: retention of heterozygosity, imbalance and $\mathrm{LOH}$. In contrast to $\mathrm{CN}$ alterations, allelic imbalance showed a significant association with local recurrence, while no association was found with distant recurrence or a combination of both. Tumors from patients in the local recurrence group showed overall statistically significant differences in allelic ratios compared to the control samples $(\mathrm{p}<0.005)$. Individual chromosome arms, and underlying segments and sub-segments, were then analyzed. Several chromosome arms showed different allelic ratio groups with (uncorrected) p-values $<0.05$ (Table 3A). In all cases, the local recurrence group displayed fewer allelic aberrations (allelic imbalance or $\mathrm{LOH}$ ) than the control group. Statistically significant sub-regions, with p-values $<0.05$ after adjustment for multiple testing, were identified on chromosome 7 . These regions showed almost no LOH (Additional file 4S4). All tumors from patients presenting with only a local recurrence showed retention of heterozygosity and balanced alleles. In contrast, approximately 50 percent of control group cases showed imbalanced alleles. The percentage of patients with retention, imbalanced alleles or $\mathrm{LOH}$ along the length of chromosome 7 is shown in Figure 2. Balanced alleles were more prominent in cases with only a local recurrence, indicating that retention on chromosome $7 \mathrm{might}$ be associated with the development of local recurrent disease. Frequent gains of chromosome 7, with or without allelic imbalances, were most often identified, and true $\mathrm{LOH}$ was almost non-existent in our study cohort. This suggests that heterozygosity of chromosome 7 is important for rectal cancer tumorigenesis and warrants further studies on the role of chromosome 7 retention in local recurrences of rectal cancer.

The use of heterozygous SNPs to determine allelic ratios of normal and tumor samples identified statistically significant differences on chromosome 7 between patients presenting with and without only local recurrent disease.

\section{Validation}

A validation of the association of chromosome 7 retention with local recurrence was performed using the 96.96 BioMark dynamic platform. A technical validation 
Table 3 Differential allelic ratio groups between local recurrence and control

\begin{tabular}{|c|c|c|c|c|c|c|c|c|}
\hline \multirow{2}{*}{$\begin{array}{l}\text { A) } \\
\text { Chr. }\end{array}$} & \multirow[b]{2}{*}{ p-value } & \multirow[b]{2}{*}{$\mathrm{BH}$} & \multicolumn{3}{|c|}{ Local recurrence group } & \multicolumn{3}{|c|}{ Control group } \\
\hline & & & Retention & Imbalance & $\overline{\mathrm{LOH}}$ & Retention & Imbalance & $\mathrm{LOH}$ \\
\hline $1 q$ & 0.05 & 0.17 & $89 \%$ & $11 \%$ & $0 \%$ & $45 \%$ & $53 \%$ & $2 \%$ \\
\hline $4 q$ & 0.05 & 0.17 & $78 \%$ & $22 \%$ & $0 \%$ & $47 \%$ & $22 \%$ & $31 \%$ \\
\hline $7 p$ & 0.0005 & 0.015 & $100 \%$ & $0 \%$ & $0 \%$ & $36 \%$ & $62 \%$ & $2 \%$ \\
\hline $7 q$ & 0.0007 & 0.015 & $100 \%$ & $0 \%$ & $0 \%$ & $9 \%$ & $13 \%$ & $78 \%$ \\
\hline $10 p$ & 0.04 & 0.17 & $89 \%$ & $11 \%$ & $0 \%$ & $53 \%$ & $45 \%$ & $2 \%$ \\
\hline $11 p$ & 0.04 & 0.17 & $89 \%$ & $11 \%$ & $0 \%$ & $49 \%$ & $42 \%$ & $9 \%$ \\
\hline $13 q$ & 0.04 & 0.17 & $78 \%$ & $11 \%$ & $11 \%$ & $27 \%$ & $60 \%$ & $13 \%$ \\
\hline $14 q$ & 0.017 & 0.17 & $78 \%$ & $22 \%$ & $0 \%$ & $40 \%$ & $22 \%$ & $38 \%$ \\
\hline $15 q$ & 0.03 & 0.17 & $78 \%$ & $22 \%$ & $0 \%$ & $29 \%$ & $29 \%$ & $42 \%$ \\
\hline $16 q$ & 0.06 & 0.18 & $89 \%$ & $11 \%$ & $0 \%$ & $53 \%$ & $36 \%$ & $11 \%$ \\
\hline $17 p$ & 0.03 & 0.17 & $22 \%$ & $33 \%$ & $44 \%$ & $9 \%$ & $13 \%$ & $78 \%$ \\
\hline $18 q$ & 0.05 & 0.17 & $11 \%$ & $22 \%$ & $67 \%$ & $9 \%$ & $13 \%$ & $78 \%$ \\
\hline $22 q$ & 0.04 & 0.17 & $78 \%$ & $11 \%$ & $11 \%$ & $33 \%$ & $31 \%$ & $36 \%$ \\
\hline \multicolumn{3}{|l|}{ B) } & \multicolumn{3}{|c|}{ Local recurrence group } & \multicolumn{3}{|c|}{ Control group } \\
\hline Chr. & \multicolumn{2}{|l|}{ p-value } & Retention & Imbalance & $\mathrm{LOH}$ & Retention & Imbalance & $\mathrm{LOH}$ \\
\hline 7 & \multicolumn{2}{|l|}{0.0025} & 9 & 0 & 0 & 19 & 25 & 1 \\
\hline 13 & \multicolumn{2}{|l|}{0.0062} & 7 & 1 & 1 & 12 & 27 & 6 \\
\hline $7+13$ & \multicolumn{2}{|l|}{$<0.0002$} & 7 & 1 & 1 & 5 & 33 & 7 \\
\hline
\end{tabular}

A) For comparison of the local recurrence group and control group, chromosome arms are listed that showed significant allelic ratio groups with $p$-values $<0.05$ and adjusted p-values $<0.2$.

Abbreviations: Chr, chromosome; B, p-value after using the Benjamin-Hochberg method for multiple testing correction.

B) For chromosome 7, chromosome 13 and in combination, the numbers of patients within each 'overall chromosome status' group - defined as the most abundant allelic ratio group on the chromosome - are shown for both the local recurrence group (L) and the control group (C). Fisher's exact test for count data was used to determine the statistical differences between analysis groups $L$ and $C$.

demonstrated that this platform could be used for validation of our results (Additional file 5 S5). We selected all (remaining) patients with a local recurrence for whom FFPE tissue was available $(\mathrm{N}=34)$. Of these patients, 22 also presented with a distant recurrence. Additionally, we included 24 patients presenting with only distant recurrences. All patients with recurrent disease were matched to control patient samples without recurrence in follow-up $(\mathrm{N}=37)$.

Differences in frequency of allelic (im)balances of specific SNPs on chromosome 7 were assessed in FFPE tissues of rectal cancer patients presenting with and without local and/or distant recurrence. Based on the results from the discovery phase, we selected 32 SNPs located on chromosome $7 \mathrm{p}(\mathrm{N}=16)$ and chromosome $7 \mathrm{q}(\mathrm{N}=16)$ (Additional file $1 \mathrm{~S} 1)$. As a high percentage of patients presenting with only a local recurrence also showed retention on chromosome 13 (Figure 2), an additional set of 16 SNPs was selected on this chromosome to potentially increase discriminative power. SNPs on both chromosome 13 and chromosome 7 were therefore included to enhance differences between analysis groups. During the discovery phase, the overall chromosome status of combined chromosomes 7 and 13 was significantly different in patients in the local recurrence group compared to the control group (Fisher's exact test for count data, p-value $<0.0002$; Table $3 \mathrm{~B}$ ). Balanced alleles were more prominent in cases with a local recurrence, indicating that retention on chromosome 7 , and to a lesser extent on chromosome 13, might be associated with the development of a local recurrence.

Using the technically validated dynamic array approach, differences between the local recurrence group and the control group showed a trend towards significance on chromosome $7 \mathrm{p}(\mathrm{p}$-value $=0.07)$. Retention of heterozygosity was more frequently observed in tumors with local recurrence compared to control group tumors, which is in accordance with findings in the discovery phase (Figure 3 ). The telomeric region of chromosome $7 \mathrm{p}$ showed allelic imbalance or $\mathrm{LOH}$ in consistently lower percentages of patients in the local recurrence group compared to the control group. For the centromeric region, the same pattern was observed for both the local recurrence group and for the local \& distant recurrence group. Chromosome 13 retention results could not be reproduced.

In brief, validation cohort data were not conclusive but do support the notion that retention of heterozygosity on the telomeric and centromeric regions of 


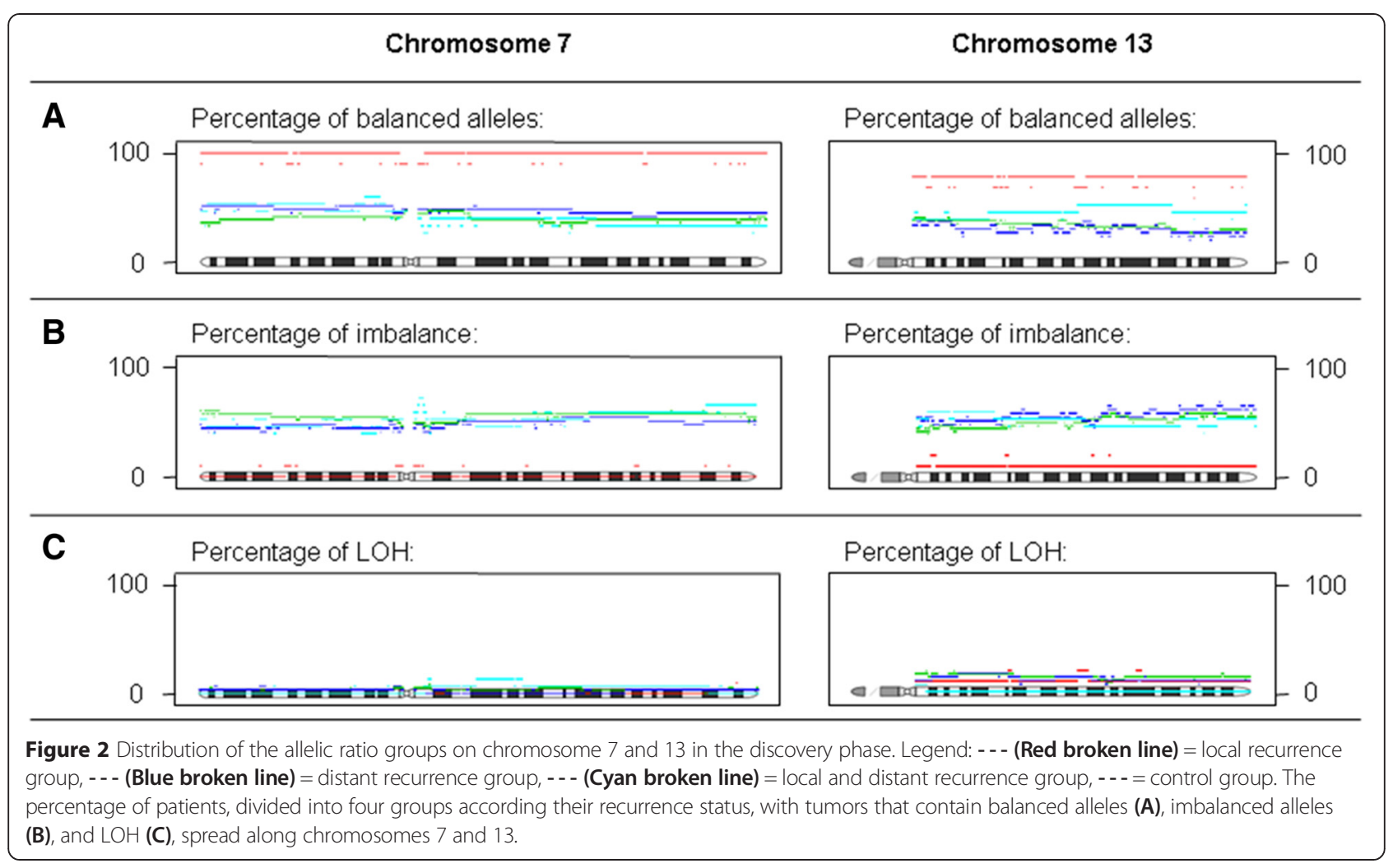

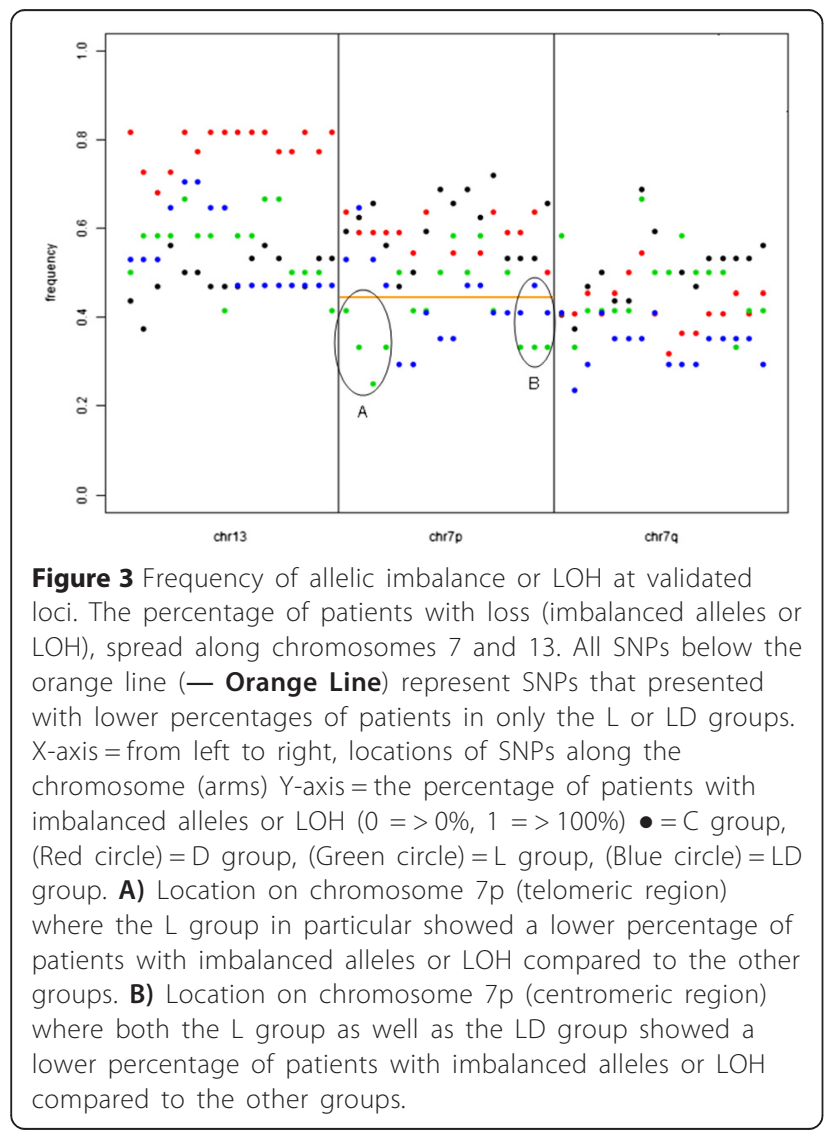

chromosome 7 may be associated with local recurrence in rectal cancer.

Prognostic value of $\mathrm{CN}$ alterations and allelic aberrations The clinical prognostic value of continuous $\mathrm{CN}$ profiles and allelic ratio group profiles was assessed in the discovery cohort using the Cox proportional hazards model. Multivariate analyses showed a trend in association of chromosome 7p with local recurrence (LRFP), but no associations with any other clinical outcome in our cohort of rectal cancer patients (Additional file 6 S6). In our discovery study cohort, allelic imbalance was associated with death by rectal cancer and local recurrent disease, with the allelic imbalance at chromosome $7 \mathrm{p}$ being chiefly responsible for the prognostic effect for local recurrent disease (Additional file 6 56). These results could not be confirmed with the 48 selected SNPs using the dynamic array (data not shown), and this outcome is reflected in the absence of literature describing associations between $\mathrm{CN}$ alterations or allelic ratio profiles and patient survival in rectal cancer patients.

Only one study specifically dedicated to rectal cancer patients reported an association of $\mathrm{CN}$ alterations with patient survival or recurrent disease [63]. The study by Doyen et al. [63] showed (in multivariate analysis) an association of loss at chromosome $8 \mathrm{p}$ with worse cancer specific survival (CSS) and the occurrence of metachronous distant metastases (DRFP). Unfortunately, the 
covariates included in the multivariate analysis where not reported. Four previously published colorectal cancer studies reported associations of $\mathrm{CN}$ alterations with patient survival or recurrent disease [11,45,48,64]. A study by De Angelis et al. [48] showed worse overall survival for patients with losses at chromosomes $1 \mathrm{p}$ and $8 \mathrm{p}$ in multivariate analyses. Bardi et al. [45] observed a significant association between loss at chromosome 4 and worse disease-free survival in univariate analyses. In multivariate analysis, loss of chromosome 18 was reported to be associated with worse overall survival. Al-Mulla et al. [11] reported that loss at chromosomes $4 \mathrm{p}$ and $5 \mathrm{q}$ was associated with worse disease-free survival in early stage colorectal cancers in multivariate analyses. Allelic imbalance at chromosome 8p was reported by Halling et al. [64] and associated with overall survival (OS) and time to recurrence (DRFP) in multivariate analysis. Association between allelic imbalance or loss at chromosome $8 \mathrm{p}$ and worse clinical outcome was described in three independent articles. However, only one was dedicated specifically to rectal cancer, and our study could not validate the results. The differences in results between these studies and our own data might be due to differences in tumor locations, as we focused on rectal cancer patients alone, whereas four out of five earlier studies included both colon and rectal cancer patients. Additionally, our use of laser capture microdissection along with macrodissection in order to reduce the potential effect of tumor microenvironment on the analyses, and thereby reduction of intratumoral heterogeneity of the rectal tumors, might provide a truer picture of the association between $\mathrm{CN}$ alterations and clinical outcome.

Allelic aberrations and $\mathrm{LOH}$ in colorectal cancer have been widely investigated, and loss of $18 \mathrm{q}$ and $17 \mathrm{p}$ are seen as prognostic markers for clinical outcomes in colorectal cancer (reviewed in [65]). To the best of our knowledge, no study has previously focused specifically on rectal cancers. A study by Choi et al. [66] showed that higher levels of $\mathrm{LOH}$ were significantly associated with tumor location specifically in the rec and the distal portion of the colon. This finding indicates that rectal and colon tumors should be considered distinct disease entities in relation to allelic alterations and $\mathrm{LOH}$ in particular. Prognostic indicators identified in studies of colorectal cancer patients cannot be adequately compared with (our) survival data on rectal cancer patients.

\section{Conclusion}

\section{Implications for clinical use}

Rectal cancer is associated with a high rate of local recurrence, in contrast to colon cancer. The location of the rectum, fixed in the smaller pelvis, provides opportunities for pRT or pCCRT treatment. Although introduction of $\mathrm{pRT}$ and $\mathrm{pCCRT}$ led to markedly reduced local recurrence rates (from $11 \%$ to $5 \%$ for pRT [2]), no difference in overall survival was observed $[2,4,8]$. This suggests that the majority of rectal cancer patients (over $90 \%$ ) are currently receiving unnecessary preoperative treatment to reduce the local recurrence risk, when in fact this risk is only relevant for less than $10 \%$ of all patients. Identification of patients who are likely to show local recurrent disease could guide decision-making for the preoperative treatment of rectal cancer patients.

Validation data from the present study on allelic ratios of chromosome 7 are supportive (but not conclusive) of our initial finding that retention of heterozygosity on chromosome 7 is associated with local recurrent disease. While these data do not yet provide sufficient grounds for development of a clinically useful platform based on these observations, further research is warranted to elucidate underlying mechanisms. Chromosome 7 harbors many interesting genes, including druggable targets such as the oncogenes EGFR, BRAF and MET, but at present their relation to the retention of chromosome 7 and the development of local recurrent disease in rectal cancer is unclear. Comparison of data on $\mathrm{CN}$ alterations and allelic imbalance from our rectal cancer cohort with previously published studies provided support for the hypothesis that colon cancer and rectal cancer may be distinct disease entities, and thus may require stratification in (survival) analyses accordingly.

\section{Additional files}

Additional file 1: Validation SNPs.

Additional file 2: Search criteria for meta-analysis.

Additional file 3: Frequency plots for $\mathrm{CN}$ gains.

Additional file 4: Statistically significant regions in allelic ratio on chromosome 7q.

Additional file 5: Technical validation of SNPs.

Additional file 6: Overall clinical prognostic value for copy number and allelic ratio.

\section{Competing interest}

The authors declare that they have no competing interests.

\section{Authors' contributions}

IJGB drafted the manuscript, participated in all aspects of the study, study design, analysed all data and performed the meta-analysis. JO analysed all data and carried out the statistical analysis. WEC participated in analysis and interpretation of CytoSNP array data. MJFWJ carried out and participated in the design and analysis of the Dynamic array experiments. BJ participated in the design of the Dynamic array experiments and in the coordination of CytoSNP array data acquisition. WW participated in the design of the Dynamic array experiments and coordination of CytoSNP array studies. ECMZ participated in sample acquisition and sample analysis. CJHVDV participated in the collection of clinical data. HM co-conceived the study, participated in the study design and sample acquisition, and maintained oversight of all aspects of the study. PJKK co-conceived the study, participated in sample acquisition and study coordination and provided supervision on all aspects of the study. TWW co-conceived and designed the study, drafted the manuscript and participated in all aspects of the study. All authors critically read, corrected and approved the final manuscript. 


\section{Acknowledgments}

This study was performed within the framework of CTMM, the Center for Translational Molecular Medicine. DeCoDe project (grant 030-101). We thank Ronald van Vlierberghe, Rob Keyzer, Melanie Schrumpf, Fahim Behrouz and Matthijs Kuppen for expert technical support.

\section{Author details}

${ }^{1}$ Department of Surgery, Leiden University Medical Center, Leiden, The Netherlands. ²Department of Pathology, L1-Q, Leiden University Medical Center, PO Box 9600, 2300 RC, Leiden, The Netherlands. ${ }^{3}$ ServiceXS,

Plesmanlaan 1d, Leiden, The Netherlands.

\section{Received: 17 December 2014 Accepted: 17 April 2015}

Published online: 06 June 2015

\section{References}

1. Kapiteijn E, Marijnen CA, Nagtegaal ID, Putter H, Steup WH, Wiggers T, et al. Preoperative radiotherapy combined with total mesorectal excision for resectable rectal cancer. N Engl J Med. 2001;345:638-46.

2. van Gijn W, Marijnen CA, Nagtegaal ID, Kranenbarg EM, Putter $H$, Wiggers T, et al. Preoperative radiotherapy combined with total mesorectal excision for resectable rectal cancer: 12-year follow-up of the multicentre, randomised controlled TME trial. Lancet Oncol. 2011;12:575-82.

3. den Dulk M, Krijnen P, Marijnen CA, Rutten HJ, van de Poll-Franse LV, Putter H, et al. Improved overall survival for patients with rectal cancer since 1990: the effects of TME surgery and pre-operative radiotherapy. Eur J Cancer. 2008:44:1710-6.

4. Yeh $\mathrm{CH}$, Chen MF, Lai CH, Huang WS, Lee SP, Chen WC, et al. Comparison of treatment results between surgery alone, preoperative short-course radiotherapy, or long-course concurrent chemoradiotherapy in locally advanced rectal cancer. Int J Clin Oncol. 2012;17:482-90.

5. Bujko K, Nowacki MP, Kepka L, Oledzki J, Bebenek M, Kryj M, et al. Postoperative complications in patients irradiated pre-operatively for rectal cancer: report of a randomised trial comparing short-term radiotherapy vs chemoradiation. Colorectal Dis. 2005;7:410-6.

6. Valentini V, Glimelius B, Haustermans K, Marijnen CA, Rodel C, Gambacorta $M A$, et al. EURECCA consensus conference highlights about rectal cance clinical management: the radiation oncologist's expert review. Radiother Oncol. 2014;110:195-8.

7. van de Velde CJ, Aristei C, Boelens PG, Beets-Tan RG, Blomqvist L, Borras JM, et al. EURECCA colorectal: multidisciplinary mission statement on better care for patients with colon and rectal cancer in Europe. Eur J Cancer. 2013:49:2784-90

8. Peeters KC, Marijnen CA, Nagtegaal ID, Kranenbarg EK, Putter H, Wiggers T, et al. The TME trial after a median follow-up of 6 years: increased local control but no survival benefit in irradiated patients with resectable rectal carcinoma. Ann Surg. 2007:246:693-701.

9. Marijnen CA, van de Velde CJ, Putter $H$, van den Brink M, Maas CP, Martijn $\mathrm{H}$, et al. Impact of short-term preoperative radiotherapy on health-related quality of life and sexual functioning in primary rectal cancer: report of a multicenter randomized trial. J Clin Oncol. 2005:23:1847-58.

10. Nilsson PJ, van EB, Hospers GA, Pahlman L, van de Velde CJ, Beets-Tan RG, et al. Short-course radiotherapy followed by neo-adjuvant chemotherapy in locally advanced rectal cancer-the RAPIDO trial. BMC Cancer. 2013;13:279.

11. Al-Mulla F, Behbehani Al, Bitar MS, Varadharaj G, Going JJ. Genetic profiling of stage I and II colorectal cancer may predict metastatic relapse. Mod Pathol. 2006;19:648-58.

12. Corver WE, Middeldorp A, ter Haar NT, Jordanova ES, van PM, van ER, et al. Genome-wide allelic state analysis on flow-sorted tumor fractions provides an accurate measure of chromosomal aberrations. Cancer Res. 2008;68:10333-40.

13. Oosting J, Lips EH, van ER, Eilers PH, Szuhai K, Wijmenga C, et al. High-resolution copy number analysis of paraffin-embedded archival tissue using SNP BeadArrays. Genome Res. 2007;17:368-76.

14. DNA isolation protocol. http://med.stanford.edu/labs/vanderijn-west/ documents/DNAextractionfromTRIZOL_Organicphase_update.doc. 2014

15. van Eijk R, Stevens $L$, Morreau $H$, van WT. Assessment of a fully automated high-throughput DNA extraction method from formalin-fixed, paraffinembedded tissue for KRAS, and BRAF somatic mutation analysis. Exp Mol Pathol. 2013:94:121-5.

16. Goeman JJ, van de Geer SA, de KF, van Houwelingen HC. A global test for groups of genes: testing association with a clinical outcome. Bioinformatics. 2004;20:93-9.
17. Goeman JJ, Oosting J, Cleton-Jansen AM, Anninga JK, van Houwelingen HC. Testing association of a pathway with survival using gene expression data. Bioinformatics. 2005;21:1950-7.

18. Benjamini Y, Hochberg Y. Controlling the False Discovery Rate: A Practical and Powerful Approach to Multiple Testing. J R Stat Soc, Series B. 1995;57:289-300,

19. Alcock HE, Stephenson TJ, Royds JA, Hammond DW. Analysis of colorectal tumor progression by microdissection and comparative genomic hybridization. Genes Chromosomes Cancer. 2003;37:369-80.

20. Ghadimi BM, Grade M, Liersch T, Langer C, Siemer A, Fuzesi L, et al. Gain of chromosome 8q23-24 is a predictive marker for lymph node positivity in colorectal cancer. Clin Cancer Res. 2003;9:1808-14.

21. He QJ, Zeng WF, Sham JS, Xie D, Yang XW, Lin HL, et al. Recurrent genetic alterations in 26 colorectal carcinomas and 21 adenomas from Chinese patients. Cancer Genet Cytogenet. 2003;144:112-8.

22. Meijer GA, Hermsen MA, Baak JP, van Diest PJ, Meuwissen SG, Belien JA, et al. Progression from colorectal adenoma to carcinoma is associated with non-random chromosomal gains as detected by comparative genomic hybridisation. J Clin Pathol. 1998;51:901-9.

23. Paredes-Zaglul A, Kang JJ, Essig YP, Mao W, Irby R, Wloch M, et al. Analysis of colorectal cancer by comparative genomic hybridization: evidence for induction of the metastatic phenotype by loss of tumor suppressor genes. Clin Cancer Res. 1998:4:879-86.

24. Ried $T$, Knutzen $R$, Steinbeck R, Blegen H, Schrock E, Heselmeyer $K$, et al. Comparative genomic hybridization reveals a specific pattern of chromosomal gains and losses during the genesis of colorectal tumors. Genes Chromosomes Cancer. 1996;15:234-45.

25. Tanami H, Tsuda H, Okabe S, Iwai T, Sugihara K, Imoto I, et al. Involvement of cyclin D3 in liver metastasis of colorectal cancer, revealed by genome-wide copy-number analysis. Lab Invest. 2005;85:1118-29.

26. Xiao XY, Zhou XY, Yan G, Sun MH, Du X. Chromosomal alteration in Chinese sporadic colorectal carcinomas detected by comparative genomic hybridization. Diagn Mol Pathol. 2007:16:96-103.

27. Chen Z, Liu Z, Li W, Qu K, Deng K, Varma MG, et al. Chromosomal copy number alterations are associated with tumor response to chemoradiation in locally advanced rectal cancer. Genes Chromosomes Cancer. 2011:50:689-99.

28. Chen Z, Liu Z, Deng X, Warden C, Li W, Garcia-Aguilar J. Chromosomal copy number alterations are associated with persistent lymph node metastasis after chemoradiation in locally advanced rectal cancer. Dis. Colon Rectum. 2012;55:677-85

29. Grade M, Becker H, Liersch T, Ried T, Ghadimi BM. Molecular cytogenetics: genomic imbalances in colorectal cancer and their clinical impact. Cell Oncol. 2006;28:71-84.

30. Grade M, Gaedcke J, Wangsa D, Varma S, Beckmann J, Liersch T. Chromosomal copy number changes of locally advanced rectal cancers treated with preoperative chemoradiotherapy. Cancer Genet Cytogenet. 2009;193:19-28.

31. Kodeda K, Asting AG, Lonnroth C, Derwinger K, Wettergren Y, Nordgren S, et al. Genomic CGH-assessed structural DNA alterations in rectal carcinoma as related to local recurrence following primary operation for cure. Int J Oncol. 2012:41:1397-404

32. Liang JW, Shi ZZ, Zhang T, Hao JJ, Wang Z, Wang XM, et al. Analysis of genomic aberrations associated with the clinicopathological parameters of rectal cancer by arraybased comparative genomic hybridization. Oncol Rep. 2013;29:1827-34

33. Lips EH, de Graaf EJ, Tollenaar RA, van ER, Oosting J, Szuhai K, et al. Single nucleotide polymorphism array analysis of chromosomal instability patterns discriminates rectal adenomas from carcinomas. J Pathol. 2007;212:269-77.

34. Lips EH, van ER, de Graaf EJ, Doornebosch PG, de Miranda NF, Oosting J et al. Progression and tumor heterogeneity analysis in early rectal cancer. Clin Cancer Res. 2008;14:772-81.

35. Molinari C, Ballardini M, Teodorani N, Giannini M, Zoli W, Emiliani E, et al. Genomic alterations in rectal tumors and response to neoadjuvant chemoradiotherapy: an exploratory study. Radiat Oncol. 2011;6:161.

36. Shi ZZ, Zhang YM, Shang L, Hao JJ, Zhang TT, Wang BS, et al. Genomic profiling of rectal adenoma and carcinoma by array-based comparative genomic hybridization. BMC Med Genomics. 2012;5:52

37. Zhou HT, Shi ZZ, Zhou ZX, Jiang YY, Hao JJ, Zhang TT, Shi F, et al. Genomic changes in rectal adenocarcinoma associated with liver metastasis. Cancer Biomark. 2013;13:281-8. 
38. Grade M, Hormann P, Becker S, Hummon AB, Wangsa D, Varma S, et al. Gene expression profiling reveals a massive, aneuploidy-dependent transcriptional deregulation and distinct differences between lymph node-negative and lymph node-positive colon carcinomas. Cancer Res. 2007;67:41-56

39. Korn WM, Yasutake T, Kuo WL, Warren RS, Collins C, Tomita M, et al. Chromosome arm 20q gains and other genomic alterations in colorectal cancer metastatic to liver, as analyzed by comparative genomic hybridization and fluorescence in situ hybridization. Genes Chromosomes Cancer. 1999;25:82-90.

40. Nakao K, Shibusawa M, Tsunoda A, Yoshizawa H, Murakami M, Kusano M, et al. Genetic changes in primary colorectal cancer by comparative genomic hybridization. Surg Today. 1998;28:567-9.

41. Schlegel J, Stumm G, Scherthan H, Bocker T, Zirngibl H, Ruschoff J, et al. Comparative genomic in situ hybridization of colon carcinomas with replication error. Cancer Res. 1995;55:6002-5.

42. Al-Mulla F, Keith WN, Pickford IR, Going JJ, Birnie GD. Comparative genomic hybridization analysis of primary colorectal carcinomas and their synchronous metastases. Genes Chromosomes Cancer. 1999;24:306-14.

43. Aragane H, Sakakura C, Nakanishi M, Yasuoka R, Fujita Y, Taniguchi H, et al. Chromosomal aberrations in colorectal cancers and liver metastases analyzed by comparative genomic hybridization. Int J Cancer. 2001;94:623-9.

44. Aust DE, Willenbucher RF, Terdiman JP, Ferrell LD, Chang CG, Moore DH, et al. Chromosomal alterations in ulcerative colitis-related and sporadic colorectal cancers by comparative genomic hybridization. Hum Pathol. 2000;31:109-14.

45. Bardi G, Fenger C, Johansson B, Mitelman F, Heim S. Tumor karyotype predicts clinical outcome in colorectal cancer patients. J Clin Oncol. 2004;22:2623-34

46. Chan TL, Curtis LC, Leung SY, Farrington SM, Ho JW, Chan AS, et al. Earlyonset colorectal cancer with stable microsatellite DNA and near-diploid chromosomes. Oncogene. 2001;20:4871-6.

47. De Angelis PM, Clausen OP, Schjolberg A, Stokke T. Chromosomal gains and losses in primary colorectal carcinomas detected by $\mathrm{CGH}$ and their associations with tumour DNA ploidy, genotypes and phenotypes. Br J Cancer. 1999;80:526-35.

48. De Angelis PM, Stokke T, Beigi M, Mjaland O, Clausen OP. Prognostic significance of recurrent chromosomal aberrations detected by comparative genomic hybridization in sporadic colorectal cancer. Int J Colorectal Dis. 2001;16:38-45

49. Diep CB, Teixeira MR, Thorstensen L, Wiig JN, Eknaes M, Nesland JM, et al. Genome characteristics of primary carcinomas, local recurrences, carcinomatoses, and liver metastases from colorectal cancer patients. Mol Cancer. 2004;3:6.

50. Georgiades IB, Curtis LU, Morris RM, Bird CC, Wyllie AH. Heterogeneity studies identify a subset of sporadic colorectal cancers without evidence for chromosomal or microsatellite instability. Oncogene. 1999;18:7933-40.

51. Hermsen M, Postma C, Baak J, Weiss M, Rapallo A, Sciutto A, et al. Colorectal adenoma to carcinoma progression follows multiple pathways of chromosomal instability. Gastroenterology. 2002;123:1109-19.

52. Knosel T, Petersen S, Schwabe H, Schluns K, Stein U, Schlag PM, et al. Incidence of chromosomal imbalances in advanced colorectal carcinomas and their metastases. Virchows Arch. 2002;440:187-94.

53. Lagerstedt KK, Kristiansson E, Lonnroth C, Andersson M, Iresjo BM, Gustafsson A, et al. Genes with relevance for early to late progression of colon carcinoma based on combined genomic and transcriptomic information from the same patients. Cancer Inform. 2010;9:79-91.

54. Nakao K, Shibusawa M, Ishihara A, Yoshizawa H, Tsunoda A, Kusano M, et al. Genetic changes in colorectal carcinoma tumors with liver metastases analyzed by comparative genomic hybridization and DNA ploidy. Cancer. 2001;91:721-6.

55. Nakao M, Kawauchi S, Uchiyama T, Adachi J, Ito H, Chochi Y, et al. DNA copy number aberrations associated with the clinicopathological features of colorectal cancers: Identification of genomic biomarkers by array-based comparative genomic hybridization. Oncol Rep. 2011;25:1603-11.

56. Poeaim S, Rerkamnuaychoke B, Jesdapatarakul S, Campiranon A Chromosome alterations in colorectal cancer in Thai patients. Cancer Genet Cytogenet. 2005;160:152-9.

57. Rooney PH, Boonsong A, McKay JA, Marsh S, Stevenson DA, Murray Gl, et al. Colorectal cancer genomics: evidence for multiple genotypes which influence survival. Br J Cancer. 2001;85:1492-8.
58. Leslie A, Pratt NR, Gillespie K, Sales M, Kernohan NM, Smith G, et al. Mutations of APC, K-ras, and p53 are associated with specific chromosomal aberrations in colorectal adenocarcinomas. Cancer Res. 2003;63:4656-61.

59. Nakao K, Mehta KR, Fridlyand J, Moore DH, Jain AN, Lafuente A, et al. Highresolution analysis of DNA copy number alterations in colorectal cancer by array-based comparative genomic hybridization. Carcinogenesis. 2004;25:1345-57.

60. Birkenkamp-Demtroder K, Olesen SH, Sorensen FB, Laurberg S, Laiho P, Aaltonen LA, et al. Differential gene expression in colon cancer of the caecum versus the sigmoid and rectosigmoid. Gut. 2005;54:374-84.

61. Komuro K, Tada M, Tamoto E, Kawakami A, Matsunaga A, Teramoto K, et al. Right- and left-sided colorectal cancers display distinct expression profiles and the anatomical stratification allows a high accuracy prediction of lymph node metastasis. J Surg Res. 2005;124:216-24.

62. Goossens-Beumer IJ, Zeestraten EC, Benard A, Christen T, Reimers MS, Keijzer R, et al. Clinical prognostic value of combined analysis of Aldh1, Survivin, and EpCAM expression in colorectal cancer. Br J Cancer. 2014;110:2935-44.

63. Doyen J, Letouze E, Marisa L, de RA, Milano G, Etienne-Grimaldi MC, et al. High-resolution analysis of DNA copy number alterations in rectal cancer: correlation with metastasis, survival, and mRNA expression. Strahlenther Onkol. 2014;190:1028-36.

64. Halling KC, French AJ, McDonnell SK, Burgart LJ, Schaid DJ, Peterson BJ, et al. Microsatellite instability and 8p allelic imbalance in stage B2 and C colorectal cancers. J Natl Cancer Inst. 1999;91:1295-303.

65. Westra JL, Plukker JT, Buys CH, Hofstra RM. Genetic alterations in locally advanced stage II/III colon cancer: a search for prognostic markers. Clin Colorectal Cancer. 2004;4:252-9.

66. Choi SW, Lee KJ, Bae YA, Min KO, Kwon MS, Kim KM, et al. Genetic classification of colorectal cancer based on chromosomal loss and microsatellite instability predicts survival. Clin Cancer Res. 2002;8:2311-22.

\section{Submit your next manuscript to BioMed Central and take full advantage of:}

- Convenient online submission

- Thorough peer review

- No space constraints or color figure charges

- Immediate publication on acceptance

- Inclusion in PubMed, CAS, Scopus and Google Scholar

- Research which is freely available for redistribution 